\title{
Haemosiderin-laden macrophages in the bronchoalveolar lavage fluid of patients with diffuse alveolar damage
}

\author{
F. Maldonado*, J.G. Parambil*, E.S. Yi”, P.A. Decker" and J.H. Ryu*
}

ABSTRACT: Quantification of haemosiderin-laden macrophages in bronchoalveolar lavage fluid (BALF) has been used to diagnose diffuse alveolar haemorrhage (DAH) but has not been assessed in patients with diffuse alveolar damage (DAD).

The present study analysed BALF obtained from 21 patients with DAD diagnosed by surgical lung biopsy.

The median age of 21 patients with DAD was 68 yrs (range 18-79 yrs); 14 (67\%) were male and 12 (57\%) were immunocompromised. The median proportion of haemosiderin-laden macrophages in BALF was $5 \%$ (range $0-90 \%$ ), but was $\geqslant 20 \%$ in seven (33\%) patients, fulfilling the commonly used BALF criterion for DAH. There was a trend toward a positive correlation between the percentage of haemosiderin-laden macrophages in BALF and parenchymal haemorrhage assessed semiquantitatively by histopathological analysis. Patients with $\geqslant 20 \%$ haemosiderinladen macrophages in BALF showed a significantly increased mortality rate $(p=0.047)$ compared to those with $<20 \%$.

In patients with an acute onset of diffuse lung infiltrates and respiratory distress, $\geqslant 20 \%$ haemosiderin-laden macrophages in BALF can occur with DAD, and is not necessarily diagnostic of DAH. The finding of $\geqslant 20 \%$ haemosiderin-laden macrophages in BALF is associated with a worse prognosis in patients with DAD.

KEYWORDS: Acute lung injury, alveolar macrophages, bronchoalveolar lavage, haemosiderin

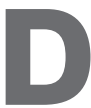

iffuse alveolar haemorrhage (DAH) is a potentially life-threatening syndrome defined by diffuse alveolar bleeding resulting from injury to the pulmonary microcirculation, such as an immune-mediated capillaritis [1-7]. DAH can be associated with various underlying disorders, including connective tissue disorders, systemic vasculitides, drugs, infections, and bone marrow or solid organ transplantation [1-7]. Patients with DAH usually present with dyspnoea and diffuse lung infiltrates, but overt indication of bleeding, i.e. haemoptysis, is not always present $[4,6]$.

Since DAH is clinically occult in some patients, bronchoalveolar lavage (BAL) has been used in diagnosing this condition, particularly in patients at excessive risk in surgical lung biopsy, e.g. thrombocytopenic or unstable patients. Several criteria based on BAL data have been proposed for diagnosing alveolar haemorrhage [8-12]. Perhaps the most commonly employed BAL criterion is the presence of $\geqslant 20 \%$ haemosiderinladen macrophages in BAL fluid (BALF) [12].
This percentage of haemosiderin-laden macrophages correlates well with the traditional Golde score $[8,9]$, a semiquantitative method of assessing the haemosiderin content of alveolar macrophages, and has been used as the diagnostic criterion of DAH in recent studies [12-14].

Diffuse alveolar damage (DAD) is a histopathological pattern of lung injury, and is the pathological correlate in most patients with acute respiratory distress syndrome (ARDS) [15-18]. Early DAD manifests an acute exudative phase that is characterised by interstitial oedema, epithelial necrosis and sloughing, the presence of fibrinous exudates in alveolar air spaces and hyaline membrane formation. In the later organising phase, resorption of hyaline membranes and intra-alveolar exudates occurs, accompanied by proliferation of type II pneumocytes along the alveolar walls and proliferation of fibroblasts in the interstitium, as well as in the airspaces $[18,19]$.

In the present study, the issue of whether increased numbers of haemosiderin-laden macrophages can be found in the BALF of
AFFILIATIONS

*Divisions of Pulmonary and Critical Care Medicine and,

"Biostatistics.

\#Dept of Laboratory Medicine and Pathology

Mayo Clinic, Rochester, MN, USA

CORRESPONDENCE

J.H. Ryu

Division of Pulmonary and Critical Care Medicine, Gonda 18 South Mayo Clinic

2001 st St SW

Rochester

MN 55905

USA

Fax: 15072664372

E-mail: ryu.jay@mayo.edu

Received

August 032008

Accepted after revision:

December 022008

STATEMENT OF INTEREST

None declared. 
patients with DAD was examined, and this finding correlated with clinicopathological features.

\section{MATERIAL AND METHODS Study subjects}

Using a computer-assisted search, 58 patients were found who had had DAD identified on surgical lung biopsy and been seen at the Mayo Clinic (Rochester, MN, USA) over a 7-yr period, January 1, 1996 to December 31, 2002. This cohort of 58 patients had been described in a previous publication [20]. Of these patients, $21(36 \%)$ had undergone BAL pre-operatively and the BALF had been analysed for haemosiderin-laden macrophages. This subset of patients formed the present study group. The Mayo Foundation Institutional Review Board approved the study.

\section{Lung biopsy}

Surgical lung biopsy specimens were obtained by limited thoracotomy in $13(62 \%)$ cases and video-assisted thoracoscopic surgery in eight (38\%). All biopsy slides were reviewed by one of the present authors (E.S. Yi), an experienced pulmonary pathologist, to confirm the diagnosis of DAD and quantify the amount of parenchymal haemorrhage and haemosiderosis, without knowledge of correlative clinical or radiological information. Parenchymal haemorrhage was histologically graded 0-3 (0: absence of intra-alveolar red blood cells; 1: questionable DAH, presence of red blood cells, but little or no fibrin; 2: intra-alveolar red blood cells and fibrin present; 3: intra-alveolar red blood cells and fibrin present in large amounts). Haemosiderosis was assessed and graded 0-3 in a similar fashion (0: no haemosiderin present; 1: questionable haemosiderosis without definite haemosiderin present; 2: haemosiderin present; 3: haemosiderin present in large amounts).

\section{Bronchoscopy}

Fibreoptic bronchoscopy was performed as previously described [21]. Sequential infusions of $20-\mathrm{mL}$ aliquots of $0.9 \%$ sodium chloride solution at $37^{\circ} \mathrm{C}$ were promptly aspirated with suction set at $80 \mathrm{mmHg}$, and collected until a total volume of $40 \mathrm{~mL}$ BAL effluent was obtained. A sample of fluid was used for bacteriological, virological and fungal studies. The remaining fluid was used for cell counts and cytological examination. Transbronchoscopic lung biopsy was performed in four $(19 \%)$ patients.

\section{Detection of haemosiderin-laden macrophages in bronchoalveolar lavage fluid}

Examination of BALF for haemosiderin-laden macrophages was performed as follows. After centrifugation (Cytospin; Shandon Southern Instruments, Runcorn, UK) for $10 \mathrm{~min}$ at $22 \times g$, a cell pellet was obtained. Perls' Prussian blue stain was used to detect haemosiderin-laden macrophages. For this purpose, air-dried slides were incubated for $10 \mathrm{~min}$ in a stain containing hydrochloric acid and potassium ferricyanide, and then counterstained with eosin and Mayer's haemalum. At a magnification of $500 \times, \geqslant 200$ alveolar macrophages were examined for the number of cells that stained with Perls' Prussian blue stain, and a percentage score was established by dividing the number of Prussian-blue-positive cells by the total number of cells counted.

\section{Statistical analysis}

Data are summarised using median, range or mean \pm SD for continuous variables, and number or percentage for categorical variables. Comparisons were performed using Fisher's exact test for categorical variables, and the two-sample rank-sum test for continuous variables. Correlation between continuous variables was assessed using Pearson's correlation coefficient. In all cases, two-tailed p-values of $\leqslant 0.05$ were considered significant.

\section{RESULTS}

\section{Demographic and clinical features}

The median age of the 21 patients was 66 yrs (range 18-79 yrs); $14(67 \%)$ were male (table 1$)$. Of these, $11(52 \%)$ had a smoking history and 12 (57\%) were immunocompromised. The causes of the immunocompromised state were haematopoietic stem cell $(n=4)$ or solid organ transplantation $(n=1)$, chemotherapy for malignancy $(n=2)$ and immunosuppressive therapy for connective tissue disorders $(n=4)$, or chronic hypersensitivity pneumonitis $(n=1)$.

All of the patients presented acutely with dyspnoea, and were admitted to the hospital. Other symptoms at presentation included cough (57\%), fever (29\%) and haemoptysis (14\%). Inspiratory crackles were heard in 19 (90\%) patients; clubbing was absent. At the time of surgical lung biopsy, 16 (76\%) patients were on invasive mechanical ventilation with lung protective strategies; the remaining five $(24 \%)$ were on noninvasive positive-pressure ventilation.

All of the patients had peripheral blood analysed for complete blood cell counts and coagulation parameters at initial presentation and serially during the course of their hospitalisation. The mean $\pm \mathrm{SD}$ haemoglobin concentration on initial evaluation was $10.6 \pm 1.6 \mathrm{~g} \cdot \mathrm{dL}^{-1}$, leukocyte count $11.1 \times 10^{3} \pm$ $7.3 \times 10^{3}$ cells $\cdot \mu \mathrm{L}^{-1}$ and platelet count $177 \times 10^{3} \pm 96 \times$ $10^{3}$ cells $\cdot \mu \mathrm{L}^{-1}$.

On the day of surgical lung biopsy, the mean arterial oxygen tension/inspiratory oxygen fraction ratio was $143.2 \pm 64.7$. Mean arterial carbon dioxide tension was $38.2 \pm 9.2 \mathrm{mmHg}$ and mean arterial pH $7.43 \pm 0.07$.

\begin{tabular}{|c|c|c|}
\hline TABLE 1 & \multicolumn{2}{|c|}{$\begin{array}{l}\text { Epidemiological aspects of } 21 \text { subjects with } \\
\text { diffuse alveolar damage }\end{array}$} \\
\hline \multicolumn{3}{|l|}{ Age yrs } \\
\hline Mean \pm SD & & $60.9 \pm 14.9$ \\
\hline Median & & 66 \\
\hline Range & & $18-79$ \\
\hline \multicolumn{3}{|l|}{ Sex n (\%) } \\
\hline Female & & 7 (33) \\
\hline Male & & $14(67)$ \\
\hline \multicolumn{3}{|c|}{ Smoking history $\mathbf{n}(\%)$} \\
\hline Current & & $10(48)$ \\
\hline Previous & & $1(5)$ \\
\hline Never & & $10(48)$ \\
\hline Immunocom & romised hosts n (\%) & $12(57)$ \\
\hline
\end{tabular}




\section{Chest radiography and computed tomography}

Mixed alveolar-interstitial infiltrates were seen bilaterally on the chest radiographs of all 21 patients. Pleural effusions were seen in two $(10 \%)$ patients, bilateral in one patient and unilateral in the other.

Computed tomography of the chest was available for all patients, and revealed a combination of consolidation and ground-glass opacities bilaterally in all patients. Other findings included pleural effusions in four (19\%) patients, which were all small in size. One patient underwent computed tomographic angiography, which was negative for pulmonary embolism.

\section{Bronchoalveolar lavage fluid analysis}

BAL was performed prior to surgical lung biopsy in all patients after a median interval of 10 days (range 3-43 days) following the onset of symptoms, and a median interval of 4 days (range 0-29 days) before surgical lung biopsy. Bronchoscopic inspection of the airways did not demonstrate gross bleeding in any patient. However, the BALF was bloodtinged or pink in $10(48 \%)$ patients. In two of these patients (central venous catheter-related sepsis and idiopathic pneumonia syndrome, respectively), the BAL return was initially clear and then became blood-tinged on sequential aliquots.

The median proportion of haemosiderin-laden macrophages in BALF was $5 \%$ (range $0-90 \%$ ) (fig. 1). The proportion of haemosiderin-laden macrophages was $\geqslant 20 \%$ in seven $(33 \%)$ patients, fulfilling the BAL criterion for the diagnosis for DAH. Four out of 10 patients with blood-tinged BALF had $\geqslant 20 \%$ haemosiderin-laden macrophages. In the remaining six patients with blood-tinged BALF, the proportion of haemosiderin-laden macrophages ranged $0-15 \%$.

On differential cell count, neutrophils were the predominant cell type identified in the BALF, with a mean percentage of $46.5 \pm 23.9$. Macrophages, lymphocytes and eosinophils accounted for $39.8 \pm 24.3,11.5 \pm 9.2$ and $2.0 \pm 2.6 \%$ of cells recovered by BAL, respectively. There was a significant

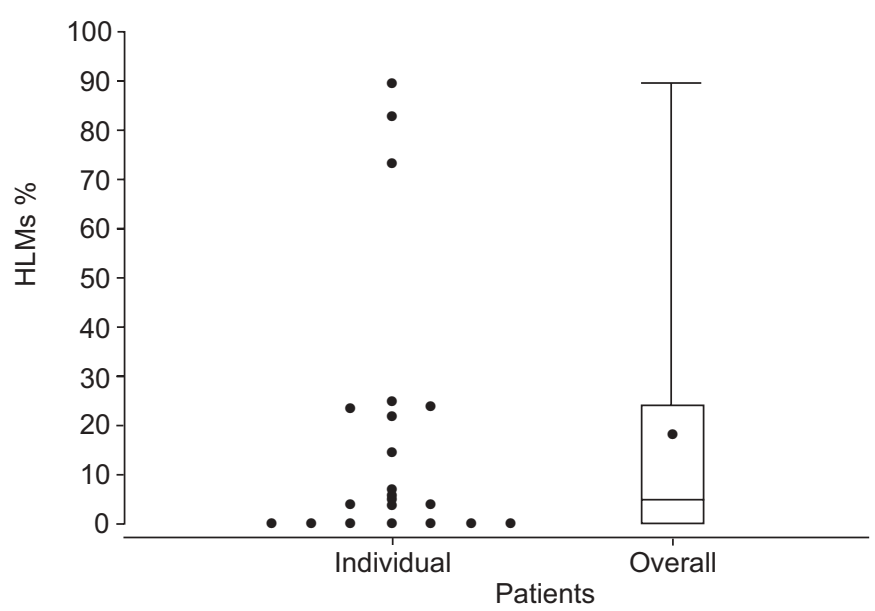

FIGURE 1. Dot plot showing the percentage of haemosiderin-laden macrophages (HLMs) in bronchoalveolar lavage fluid from 21 patients with diffuse alveolar damage. The box represents median and interquartile range; the single point represents the mean (SD 28.26) and the vertical bars range. negative correlation between the percentage of haemosiderinladen macrophages and the percentage of neutrophils in BALF $(\mathrm{r}=-0.62 ; \mathrm{p}=0.003$ (Pearson's correlation coefficient)). There was a positive correlation between the percentage of haemosiderin-laden macrophages and total percentage of macrophages in BALF $(r=0.64 ; p=0.002$ (Pearson's correlation coefficient)).

Transbronchoscopic lung biopsy was performed in four (19\%) patients. Histopathological examination of these biopsy specimens revealed features of organising pneumonia in one patient, DAD in one and were nondiagnostic in the remaining two.

\section{Surgical lung biopsy}

Surgical lung biopsy was performed to clarify the aetiology of persistent lung infiltrates and associated respiratory insufficiency. Histopathological features of DAD were demonstrated in all of the patients. The median interval from initial presentation to surgery was 12 days (range 3-56 days). Lung specimens were obtained by limited thoracotomy in $13(62 \%)$ patients, and by video-assisted thoracoscopic surgery in eight $(38 \%)$. None of the patients had evidence of pulmonary capillaritis. Four out of 21 patients showed areas of grade 3 haemorrhage, which involved relatively limited patchy foci. Three out of 21 cases revealed grade 3 haemosiderosis, which was also seen in only a few small areas. Additional histopathological findings included superimposed acute bronchopneumonia $(n=4)$, usual interstitial pneumonia $(n=3)$, amyloid $(n=1)$ and necrotising granulomas $(n=1)$.

There was no significant correlation between the percentage of haemosiderin-laden macrophages in BALF and semiquantitative assessment of parenchymal haemosiderosis on histopathological analysis $(\mathrm{r}=0.16 ; \mathrm{p}=0.487)$. Although nonsignificant, there was a trend toward a positive correlation between the percentage of haemosiderin-laden macrophages in BALF and semiquantitative assessment of parenchymal haemorrhage on histopathological analysis $(r=0.42 ; \mathrm{p}=0.061)$.

\section{Causes of diffuse alveolar damage}

The underlying cause of DAD was identifiable in $17(81 \%)$ patients, and included infections in five (24\%), drug-induced pulmonary toxicity in four (19\%), idiopathic pulmonary fibrosis (acute exacerbation) in three (14\%), noninfectious pulmonary complications of haematopoietic stem cell or solid organ transplantation in three $(14 \%)$, and chronic hypersensitivity pneumonitis in one $(5 \%)$. The remaining patient had mixed connective tissue disease, which was thought to be the cause of the DAD. In four (14\%) cases, the underlying cause was not directly identifiable, i.e. acute interstitial pneumonia.

\section{Subset analysis of patients with $\geqslant 20 \%$ haemosiderin-laden} macrophages in bronchoalveolar lavage fluid

As already noted, seven patients had $\geqslant 20 \%$ haemosiderinladen macrophages in BALF, with values ranging 22-90\% (median 25\%). The underlying causes of DAD and correlative findings of these seven patients are listed in table 2. One of these patients (74-yr-old male) had been on chronic anticoagulant therapy for a prosthetic mitral valve; anticoagulant therapy was discontinued 2 days before bronchoscopy with a prothrombin time international normalised ratio of 1.6 on the 
day of BAL. Two other patients (57 and 75-yr-old males) were thrombocytopenic, with platelet counts of $36 \times 10^{9}$ and $67 \times 10^{9}$ cells $\cdot \mathrm{L}^{-1}$, respectively, on the day of BAL. These three patients accounted for those with the highest haemosiderinladen macrophage percentages. However, two out of 14 patients with $<20 \%$ haemosiderin-laden macrophages in BALF were also thrombocytopenic (platelets $<50 \times$ $10^{9}$ cells $\cdot \mathrm{L}^{-1}$ ) at the time of their BAL. No other patient was known to have any bleeding diathesis.

Comparison of these seven patients with the 14 remaining patients who had $<20 \%$ haemosiderin-laden macrophages in BALF revealed no difference in the interval between onset of symptoms and bronchoscopy or surgical lung biopsy. All seven patients with $\geqslant 20 \%$ haemosiderin-laden macrophages in BALF died during their hospitalisation versus only seven $(50 \%)$ of those with $<20 \%$ haemosiderin-laden macrophages, a difference that was significant $(p=0.047$ (Fisher's exact test)).

\section{DISCUSSION}

In the present study of 21 patients with DAD diagnosed by surgical lung biopsy, a third had $\geqslant 20 \%$ haemosiderin-laden macrophages in BALF, a criterion commonly used to diagnose DAH [12-14, 22]. The present findings suggest that diagnosis of DAH that is based on the percentage of haemosiderin-laden macrophages in BALF may be inaccurate. This is problematic since the clinical presentation and radiological features of DAD and DAH are similar [23].

$\mathrm{DAH}$ is histologically characterised by widespread intraalveolar haemorrhage that can be both acute and chronic. The vast majority of cases of DAH are thought to be secondary to capillaritis of immune or non-immune causes [1, 4-7, 24]. Although often regarded as synonymous with DAH, capillaritis is a pathological diagnosis defined by the presence of neutrophils infiltrating the capillary walls with occasional fibrinoid necrosis of the alveolar wall and secondary destruction of the lung architecture [1, 5]. Bland pulmonary haemorrhage (without capillaritis) may also result in $\mathrm{DAH}$, and most often results from blood dyscrasia [5]. Other conditions associated with pulmonary haemorrhage, such as Goodpasture's syndrome and systemic lupus erythematosus, do not usually show significant pulmonary capillaritis. In such cases, the pathological findings may resemble DAD, especially in the exudative phase. Conversely, DAD may be associated with increased intra-alveolar red blood cells, fibrin and haemosiderin, which could occasionally mimic DAH. The absence of capillaritis could be helpful, but careful clinical correlation may be needed to distinguish DAD from DAH without capillaritis in some cases.

Several bronchoscopic diagnostic criteria have been proposed for the diagnosis of DAH but have not been systematically validated against pathological data [8, 10-12]. Surgical biopsy examination is considered the gold standard but is often impractical. Clues to true alveolar haemorrhage include the presence of haemosiderin in the interstitium, fibrin entangled with red blood cells and haemosiderin-laden macrophages [5]. The mere presence of intra-alveolar red blood cells is not diagnostic as it may result from surgical trauma, as is often the case in bronchoscopic lung biopsy.

Bronchoscopic alveolar lavage has been accepted as a minimally invasive procedure for establishing the diagnosis of DAH [12-14]. GOLDE et al. [8] described the Golde score, which assigned a rank to the haemosiderin content of alveolar macrophages based on a subjective estimate after examining 200 alveolar macrophages (0: no colour; 1 : faint blue in one portion of the cytoplasm; 2 : deep blue in a minor portion of the cell; 3: deep blue in most areas of the cytoplasm; 4: deep blue throughout the cell). The total score from 200 cells was divided by 2 in order to obtain a haemosiderin score for a mean of 100 alveolar macrophages. A Golde score of $>100$ was considered severe DAH, whereas a score of $20-100$ was considered mildto-moderate DAH. This original study consisted of five leukaemic patients, one of whom had histopathological confirmation (autopsy) of pulmonary haemorrhage.

DE LASSENCE et al. [12] noted a close correlation between the Golde score and the percentage of haemosiderin-laden macrophages in BALF identified by Perl's Prussian blue staining and suggested the use of this percentage since it was easier to perform. Thus, $\geqslant 20 \%$ haemosiderin-laden macrophages is commonly regarded as diagnostic of DAH; however, direct correlation to histopathological data has been lacking.

\begin{tabular}{|c|c|c|c|c|c|c|c|c|}
\hline \multicolumn{2}{|c|}{ TABLE 2} & \multicolumn{7}{|c|}{$\begin{array}{l}\text { Summary of patients with diffuse alveolar damage and } \geqslant 20 \% \text { haemosiderin-laden macrophages in bronchoalveolar } \\
\text { lavage fluid }\end{array}$} \\
\hline $\begin{array}{l}\text { Patient } \\
\text { No. }\end{array}$ & $\begin{array}{l}\text { Age } \\
\text { yrs }\end{array}$ & Sex & Diagnosis & BAL site & HLMs \% & $\begin{array}{l}\text { BAL-biopsy } \\
\text { interval days }\end{array}$ & SLB site & $\begin{array}{l}\text { Haemorrhage } \\
\text { score }^{\#}\end{array}$ \\
\hline 1 & 48 & M & Cocaine-induced & Lingula & 22 & 29 & LLL & 1 \\
\hline 2 & 74 & M & Acute exacerbation of IPF & RML & 74 & 6 & LUL/LLL & 3 \\
\hline 4 & 75 & M & Herpes simplex virus type1 pneumonia & RUL & 24 & 4 & RUL/RLL & 2 \\
\hline 5 & 75 & M & Acute exacerbation of IPF & RML & 24 & 12 & RUL/RLL & 2 \\
\hline 6 & 68 & M & Central venous catheter-related sepsis (multiple myeloma) & RML & 90 & 3 & RUL/RLL & 2 \\
\hline 7 & 68 & $\mathrm{~F}$ & Cytarabine-induced (acute myelogenous leukaemia) & RUL & 25 & 2 & $\mathrm{RML/RLL}$ & 1 \\
\hline
\end{tabular}

BAL: bronchoalveolar lavage; HLM: haemosiderin-laden macrophage; SLB: surgical lung biopsy; IPF: idiopathic pulmonary fibrosis; LLL: left lower lobe; LUL: left upper

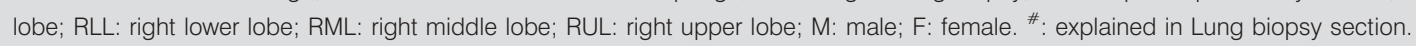


DAD is a relatively common histopathological finding on lung biopsy or at autopsy $[15,17,20]$. It is the most common histology seen in patients with ARDS $[15,17]$. DAD represents an evolving process of severe injury to the alveolar-capillary units [15, 17]. In particular, an earlier exudative phase is associated with interstitial and alveolar oedema resulting from disrupted integrity of the alveolar-capillary structures. Hyaline membranes form and intra-alveolar haemorrhage can be seen. In addition, microthrombi, as well as larger thromboemboli and haemorrhagic infarcts, are commonly seen in patients with DAD $[15,17]$. Thus it is not unexpected that DAD can be associated with increased numbers of haemosiderin-laden macrophages in BALF.

Nearly all (95\%) of the present patients with DAD showed BALF neutrophilia ( $>3 \%$ neutrophils). This is consistent with the underlying process of acute lung injury. The percentage of haemosiderin-laden macrophages was also noted to correlate in a positive manner with the total percentage of BALF macrophages, and negatively with the percentage of BALF neutrophils. Macrophages are thought to play an important role in the progression of DAD towards the fibroproliferative phase, and intra-alveolar neutrophils have been associated with membrane repair and remodelling [18, 19]. Since the number of subjects in the present study was relatively small, it is unclear whether or not these correlations represent a valid observation. Results opposite from those observed here, i.e. a positive correlation between the percentage of haemosiderinladen macrophages and the percentage of neutrophils, might perhaps have been expected if the latter were reflective of the underlying inflammatory activity and alveolar-capillary injury. Another potential confounder in attempting to make sense of these correlations is the fact that BAL was performed at different time points in the evolving process of DAD.

No significant correlation was noted between BAL findings and haemorrhage and haemosiderin scores as determined in surgical biopsy specimens. One possible explanation for this is the varying interval between bronchoscopic sampling and surgical biopsy among these 21 patients, which may have obscured the correlation in the temporally evolving process of DAD $[25,26]$. The site of surgical lung biopsy was, in some cases, different from the lobe in which BAL was performed. It is suspected that the degree of parenchymal haemorrhage present on lung biopsy would have correlated better with the percentage of haemosiderin-laden macrophages in BALF had BAL been performed near the time of surgical lung biopsy and in the same location. This inconsistency in the location and timing of BAL and lung biopsy probably also contributed to the lack of correlation between the percentage of haemosiderin-laden macrophages in BALF and semiquantitative assessment of parenchymal haemosiderosis on histopathological analysis. Complete clearance of haemosiderin from the lung has been observed to occur within 2-4 weeks following acute pulmonary haemorrhage $[12,25]$. A larger and prospective study with a design addressing these shortcomings is needed in order to further clarify this issue.

The present results suggest that an increased percentage of haemosiderin-laden macrophages in BALF may be of prognostic significance as all patients with $\geqslant 20 \%$ haemosiderinladen macrophages died during their hospitalisation. This correlation is probably due to higher percentages of haemosiderin-laden macrophages reflecting more extensive acute lung injury. In this regard, it is interesting to note that ABU-FARSAKH et al. [27] noted better prognosis to be associated with the absence of haemosiderin-laden macrophages in BALF among bone marrow transplant patients.

The present study has limitations due to its retrospective nature and limited number of patients. However, the results raise important questions that should be addressed in larger prospective studies. Based on the present data, it appears that DAD could be mistaken for DAH when the latter diagnosis is based upon the percentage of haemosiderin-laden macrophages in BALF. Coexisting bleeding diathesis, such as thrombocytopenia or recent anticoagulant therapy, may contribute to elevating the percentage of haemosiderin-laden macrophages in the BALF of patients with DAD, as seen in three of the present patients with the highest counts. Corticosteroid therapy is commonly employed in the management of patients with DAH, but its role in the treatment of DAD remains unclear. In spite of fairly extensive data on the use of corticosteroids in ARDS in the literature, no consensus as to their role has been established. Although they may offer benefit in some specific clinical contexts, they could be potentially harmful in others [28, 29]. Thus accurate diagnostic separation of DAH and DAD may have management implications.

In conclusion, the present results suggest that an increased percentage of haemosiderin-laden macrophages in BALF can be seen in patients with DAD, and is not specific for DAH. Patients with $\geqslant 20 \%$ haemosiderin-laden macrophages in BALF have a worse prognosis than those with $<20 \%$. Further studies analysing the diagnostic and prognostic implications of BAL results in patients with DAD are warranted.

\section{REFERENCES}

1 Green RJ, Ruoss SJ, Kraft SA, et al. Pulmonary capillaritis and alveolar hemorrhage. Update on diagnosis and management. Chest 1996; 110: 1305-1316.

2 Dweik RA, Arroliga AC, Cash JM. Alveolar hemorrhage in patients with rheumatic disease. Rheum Dis Clin North Am 1997; 23: 395-410.

3 Zamora MR, Warner ML, Tuder R, et al. Diffuse alveolar hemorrhage and systemic lupus erythematosus. Clinical presentation, histology, survival, and outcome. Medicine 1997; 76: 192-202.

4 Specks U. Diffuse alveolar hemorrhage syndromes. Curr Opin Rheumatol 2001; 13: 12-17.

5 Colby TV, Fukuoka J, Ewaskow SP, et al. Pathologic approach to pulmonary hemorrhage. Ann Diagn Pathol 2001; 5: 309-319.

6 Schwarz MI, Fontenot AP. Drug-induced diffuse alveolar hemorrhage syndromes and vasculitis. Clin Chest Med 2004; 25: 133-140.

7 Collard HR, Schwarz MI. Diffuse alveolar hemorrhage. Clin Chest Med 2004; 25: 583-592.

8 Golde DW, Drew WL, Klein HZ, et al. Occult pulmonary haemorrhage in leukaemia. BMJ 1975; 2: 166-168.

9 Finley TN, Aronow A, Cosentino AM, et al. Occult pulmonary hemorrhage in anticoagulated patients. Am Rev Respir Dis 1975; 112: 23-29. 
10 Grebski E, Hess T, Hold G, et al. Diagnostic value of hemosiderin-containing macrophages in bronchoalveolar lavage. Chest 1992; 102: 1794-1799.

11 Kahn FW, Jones JM, England DM. Diagnosis of pulmonary hemorrhage in the immunocompromised host. Am Rev Respir Dis 1987; 136: 155-160.

12 De Lassence A, Fleury-Feith J, Escudier E, et al. Alveolar hemorrhage. Diagnostic criteria and results in 194 immunocompromised hosts. Am J Respir Crit Care Med 1995; 151: 157-163.

13 Lauque D, Cadranel J, Lazor R, et al. Microscopic polyangiitis with alveolar hemorrhage. A study of 29 cases and review of the literature. Groupe d'Etudes et de Recherche sur les Maladies "Orphelines" Pulmonaires (GERM“'O"P). Medicine 2000; 79: 222-233.

14 Afessa B, Tefferi A, Litzow MR, et al. Diffuse alveolar hemorrhage in hematopoietic stem cell transplant recipients. Am J Respir Crit Care Med 2002; 166: 641-645.

15 Tomashefski JF Jr. Pulmonary pathology of acute respiratory distress syndrome. Clin Chest Med 2000; 21: 435-466.

16 Esteban A, Fernandez-Segoviano P, Frutos-Vivar F, et al. Comparison of clinical criteria for the acute respiratory distress syndrome with autopsy findings. Ann Intern Med 2004; 141: 440-445.

17 Castro CY. ARDS and diffuse alveolar damage: a pathologist's perspective. Semin Thorac Cardiovasc Surg 2006; 18: 13-19.

18 Dos Santos CC. Advances in mechanisms of repair and remodelling in acute lung injury. Intensive Care Med 2008; 34: 619-630.

19 Dechert RE. The pathophysiology of acute respiratory distress syndrome. Respir Care Clin N Am 2003; 9: 283-296.
20 Parambil JG, Myers JL, Aubry MC, et al. Causes and prognosis of diffuse alveolar damage diagnosed on surgical lung biopsy. Chest 2007; 132: 50-57.

21 Cordonnier C, Bernaudin JF, Fleury J, et al. Diagnostic yield of bronchoalveolar lavage in pneumonitis occurring after allogeneic bone marrow transplantation. Am Rev Respir Dis 1985; 132: 1118-1123.

22 Costabel U, Guzman J. Bronchoalveolar lavage in interstitial lung disease. Curr Opin Pulm Med 2001; 7: 255-261.

23 Schwarz MI, Albert RK. "Imitators" of the ARDS: implications for diagnosis and treatment. Chest 2004; 125: 1530-1535.

24 Travis WD, Colby TV, Lombard C, et al. A clinicopathologic study of 34 cases of diffuse pulmonary hemorrhage with lung biopsy confirmation. Am J Surg Pathol 1990; 14: 1112-1125.

25 Sherman JM, Winnie G, Thomassen MJ, et al. Time course of hemosiderin production and clearance by human pulmonary macrophages. Chest 1984; 86: 409-411.

26 Epstein CE, Elidemir O, Colasurdo GN, et al. Time course of hemosiderin production by alveolar macrophages in a murine model. Chest 2001; 120: 2013-2020.

27 Abu-Farsakh HA, Katz RL, Atkinson N, et al. Prognostic factors in bronchoalveolar lavage in 77 patients with bone marrow transplants. Acta Cytol 1995; 39: 1081-1088.

28 Steinberg KP, Hudson LD, Goodman RB, et al. Efficacy and safety of corticosteroids for persistent acute respiratory distress syndrome. N Engl J Med 2006; 354: 1671-1684.

29 Peter JV, John P, Graham PL, et al. Corticosteroids in the prevention and treatment of acute respiratory distress syndrome (ARDS) in adults: meta-analysis. BMJ 2008; 336: 1006-1009. 